\title{
Escala de Percepção do Suporte Social - versão Adulta: um estudo pela Teoria de Resposta ao Item
}

\author{
Scale of Perceived Social Support - Adult version: \\ A study using Item Response Theory
}

Hugo Ferrari CARDOSO ${ }^{1}$

Makilim Nunes BAPTISTA2

\section{Resumo}

A presente pesquisa teve por objetivo verificar os parâmetros dos itens e das pessoas, obtidos por meio do modelo de Rasch, para os fatores da Escala de Percepção do Suporte Social - versão Adulta. A amostra foi composta por 533 universitários, provenientes de três estados brasileiros, com idade entre 18 e 62 anos, sendo a maioria do sexo feminino (69\%). Os participantes responderam à Escala de Percepção do Suporte Social - versão Adulta, composta por 36 itens, distribuídos em quatro dimensões: Afetivo; Interações Sociais; Instrumental; Enfrentamento de Problemas. Os resultados encontrados apontaram a adequação do instrumento, com base no modelo de Rasch. Todos os itens da Escala estiveram dentro dos parâmetros recomendados pelo modelo, assim como as categorias de resposta se mostraram discriminativas. Pôde-se constatar que a média do mapa de itens esteve abaixo da média das pessoas, indicando que eles foram facilmente endossados pela amostra.

Palavras-chave: Psicometria; Suporte social; Teoria de resposta ao item.

\begin{abstract}
This study aimed to verify the parameters of the items and persons using the Rasch model for the factors of the Scale of Perceived Social Support (adult version). The sample consisted of 533 students from three Brazilian states, with ages ranging from 18 to 62 years, the majority being female (69\%). Participants responded to the Scale of Perceived Social Support (adult version), consisting of 36 items, divided into four elements, namely Affective; Social Interactions; Instrumental; and Coping with Problems. The results indicated the suitability of the elements of the Scale of Perceived Social Support (adult version), based on the Rasch model. All scale items were within the parameters recommended by the model, and the categories of responses proved to be discriminative. The average of the items from the item map was below the average for people, indicating that the items were easily endorsed by the sample.
\end{abstract}

Keywords: Psychometrics; Social support; Item response theory.

$\nabla \nabla \nabla v$

1 Universidade Estadual Paulista Júlio de Mesquita Filho, Faculdade de Ciências, Departamento de Psicologia. R. Eng. Luiz Edmundo Carrijo Coube, 14-01, Vargem Limpa, 17033-360, Bauru, SP, Brasil. Correspondência para/Correspondence to: H.F. CARDOSO. E-mail: $<$ hfcardoso@gmail.com>.

2 Universidade São Francisco, Departamento de Psicologia, Programa de Pós-Graduação em Psicologia. Itatiba, SP, Brasil. 
A construção de um instrumento psicológico implica uma série de procedimentos até o estabelecimento de normas e redação do manual, ou seja, há a necessidade de buscar propriedades psicométricas que atestem sua funcionalidade (Werlang, Villemor-Amaral, \& Nascimento, 2010). De acordo com Carvalho (2011), é comum os instrumentos serem construídos a partir de pressupostos advindos da Teoria Clássica dos Testes (TCT), ou seja, com parâmetros de validade (por meio de evidência de validade, com base no conteúdo, no processo de resposta, na estrutura interna, na relação com variáveis externas; ou evidências baseadas nas consequências da testagem) e parâmetros de precisão (teste-reteste, interavaliadores, forma alternada, duas metades e consistência interna).

De acordo com Pasquali e Primi (2007), o modelo da TCT é dependente da amostra à qual o instrumento psicológico é aplicado e, nesse sentido, sua validade pode ser contestada na medida em que se altera o tipo de amostra. Hambleton, Swaminathan e Rogers (1991) e Pasquali (2007) atentaram para o fato de que, por intermédio da TCT, os parâmetros psicométricos dos itens também são dependentes da amostra e, dessa forma, os itens podem ser mais fáceis ou difíceis de serem respondidos tendo como parâmetro apenas a habilidade dos sujeitos respondentes. Ainda, Vieira, Ribeiro, Almeida e Primi (2011) complementam que, por meio da TCT, um teste de fácil resolução possuirá propriedades para avaliação de pessoas com baixa aptidão, enquanto um teste difícil avaliará bem apenas as pessoas com aptidões elevadas.

Além das questões até então levantadas, Hambleton e van der Linden (1982) atentam para os índices de fidedignidade de um instrumento quando se faz uso da TCT. De acordo com os autores, nesses parâmetros, a confiabilidade é obtida por um índice geral, sem se considerar a relação entre dificuldades dos itens e as características da amostra. Nessa mesma direção, Wiberg (2004) aponta limitações da TCT no que tange a algumas análises pertinentes ao processo de construção e adequação de instrumentos, destacando que a 468 Teoria de Resposta ao Item (TRI) pode ser uma alter- nativa para sanar algumas dessas dificuldades metodológicas.

De acordo com Hambleton et al. (1991), a TRI apresenta algumas vantagens quando comparada à TCT. A primeira é a independência dos itens em relação à amostra, pois, nesse modelo, as aptidões dos indivíduos podem ser calculadas por itens isolados, e não pelo seu conjunto. Além disso, é possível verificar o emparelhamento dos itens em relação à aptidão do indivíduo, o que proporciona uma avaliação mais precisa - como destacam Andrade e Valle (1998) e Vieira et al. (2011) -, por adequar as dificuldades de um item ao nível de aptidão dos sujeitos.

Embora a Teoria Clássica dos Testes seja importante instrumento de avaliação psicológica (Carvalho, 2011), a TRI surge, conforme aponta Muñiz (2010), na medida em que os pesquisadores passaram a constatar que o modelo apresentava deficiências no alcance de algumas análises. Para esse autor, trata-se de dois modelos diferentes. Enquanto a TCT tem o foco de estudo no teste e pode ser bem realizada em uma amostra de até 500 pessoas, a TRI apresenta o foco de estudo centrado no item do instrumento e é recomendada para um número amostral superior àquele. No entanto, este último número não é consensual, conforme ressaltam Valentini e Laros (2011).

Tais autores, citando outros estudos, informam que para a utilização do modelo da TRI, Comrey e Lee (1992) sugerem um número entre 100 e 300 participantes, enquanto Nunes e Primi (2005) apontam amostra superior a 200 sujeitos. Já de acordo com Reise, Ainsworth e Haviland (2005), a TRI é um modelo de análise pautado em cálculos matemáticos e procedimentos estatísticos.

Os precursores da TRI, já em meados da década de 1950, buscavam um novo modelo para sanar as lacunas percebidas na utilização da TCT. Além disso, as inovações tecnológicas, principalmente computacionais, proporcionaram verdadeiros avanços a esse modelo, com softwares específicos para execução das complexas operações matemáticas utilizadas na TRI (Conde \& Laros, 2007; Pasquali \& Primi, 2003; Pasquali \& Primi, 2007; Primi, 2004; Vendramini, 2005). 
Com a utilização da TRl, é possível buscar informações que contribuem no processo de validação de instrumentos psicológicos (Valentini \& Laros, 2011). De acordo com Carvalho e Primi (2010) e Linacre (2009), por meio de um procedimento de normatização do item, é possível realizar inferências com base nas pontuações obtidas nos itens de um teste psicológico, em relação aos padrões de resposta esperados. Conforme Embretson e Reise (2000), pela TRI, são estimados os traços latentes, ou theta $(\theta)$, referentes à probabilidade de acerto, ou endosso, dos indivíduos em relação ao conteúdo dos itens.

Nesse sentido, no caso de um teste psicológico de percepção de determinado construto, tendo como opção de resposta o modelo Likert, ou seja, politômico, um item será mais fácil ou difícil de ser respondido dependendo da intensidade do traço latente nesses sujeitos. Em outras palavras, quanto mais intensa for determinada característica em uma pessoa, maior é a probabilidade de endosso em itens que avaliam tais conteúdos, e vice-versa (Pasquali \& Primi, 2007).

Embretson (2006) ressaltou que há vários modelos em TRI, sendo o modelo de Rasch um dos mais utilizados. Tal procedimento, também denominado "modelo de um parâmetro", avalia o item com base em seu nível de dificuldade, conhecido como parâmetro "b". Valentini e Laros (2011) ponderam que um dos pressupostos da TRI é a unidimensionalidade, ou seja, o conjunto de itens deve avaliar apenas um traço latente, o que representa certa dificuldade, uma vez que, em geral, as escalas psicológicas tendem a ter os itens distribuídos em fatores, como ocorre com a Escala de Percepção do Suporte Social - versão Adulta (EPSUS-A). Os autores comentam ainda que é possível, por meio da TRI, executar análise com diversos fatores, desde que sejam cumpridos dois critérios. O primeiro consiste em realizar uma análise fatorial prévia, por meio da TCT, comprovando estatisticamente as dimensões; enquanto o segundo, pela TRI, deve considerar os itens separados em seus fatores, sem tomar o teste como um conjunto.

A propósito, a EPSUS-A é um instrumento psicológico apto a avaliar a percepção do suporte social; possui evidência de validade com base na estrutura interna, mediante análise fatorial, que, por meio da TCT, confirmou quatro fatores relacionados ao construto-alvo (Cardoso, 2013). Com base nisso, o presente estudo, fazendo uso da TRI, buscou verificar os parâmetros dos itens e das pessoas, obtidos por meio do modelo de escala graduada para a EPSUS-A, a fim de evidenciar outra validade com base na estrutura interna. Especificamente, foram estimados os parâmetros dos itens do teste e dos participantes do estudo. Verificou-se que os parâmetros encontrados correspondiam ao que era esperado conforme o modelo matemático. Também foram estimados os índices de correlação e fidedignidade, assim como se analisaram as categorias de resposta das escalas e o mapa de itens-pessoas, em cada dimensão da EPSUS-A.

\section{Método}

\section{Participantes}

A amostra da pesquisa foi composta por 533 estudantes que cursavam o ensino superior, tanto na graduação $(n=443)$ quanto na pós-graduação $(n=90)$. Os participantes eram provenientes dos estados de São Paulo ( $n=438)$, Minas Gerais $(n=68)$ e Bahia $(n=27)$ e situavam-se na faixa etária entre 18 e 62 anos ( $M=25,6$; $D P=8,2)$, sendo a maioria (368) do sexo feminino (69\%).

\section{Instrumentos}

Foi utilizado a Escala de Percepção do Suporte Social - versão Adulta (Baptista \& Cardoso, 2012) que tem como objetivo avaliar a percepção do suporte social. Tal construto pode ser entendido como a percepção, por parte do indivíduo, de que ele possui, em sua rede social, pessoas que Ihe proporcionam apoio em determinados momentos de sua vida, sejam eles de dificuldade ou não. Inicialmente o instrumento era composto por 77 itens, os quais, após realização da análise fatorial, foram reduzidos para 36 e distribuídos em 4 dimensões: Afetivo (17 itens e índice de precisão alfa de Cronbach 
$\alpha=0,92$ ), Interações Sociais (5 itens e $\alpha=0,75$ ), Instrumental (7 itens e $\alpha=0,82$ ) e Enfrentamento de Problemas (7 itens e $\alpha=0,83$ ).

O fator 1 (Afetivo) é composto por itens relacionados ao suporte de ordem emocional, que o indivíduo percebe poder receber de outras pessoas, como por exemplo, "Posso contar com pessoas que me proporcionam situações agradáveis".

Já o fator 2 (Interação social) possui itens relativos ao relacionamento do sujeito com outros indivíduos, bem como à possibilidade de participação em eventos sociais, como exemplo "Posso contar com pessoas que (a) me convidam para atividades sociais".

No fator 3 (Instrumental) agrupam-se itens referentes à percepção de suporte de ordem material, tais como "Posso contar com pessoas que (a) pagam minhas contas quando tenho um problema financeiro".

Já o fator 4 (Enfrentamento de problemas) abrange itens referentes à circulação de informação nas interações sociais, assim como à percepção de pessoas concebidas como suportivas, que oferecem conselhos e instruções úteis na tomada de decisão e na resolução de conflitos, como pode ser percebido no item "Posso contar com pessoas que (a) compreendem meus problemas".

A Escala de Percepção do Suporte Social - versão Adulta obedece a uma escala Likert de 4 pontos (Sempre; Muitas vezes; Poucas vezes; Nunca), com pontuação variando entre 0 e 108; quanto maior a pontuação na escala, maior a percepção de suporte social.

\section{Procedimentos}

O projeto foi aprovado pelo Comitê de Ética em Pesquisa da universidade em que se realizou a pesquisa (Protocolo nº 0240.0.142.000-11). Após assinatura do Termo de Consentimento Livre e Esclarecido (TCLE), a EPSUS-A foi aplicada aos universitários, em sala de aula com média de 40 alunos, por cerca de 15 minutos e conforme a disponibi470 lidade dos professores.

\section{Plano de análise de dados}

Tendo a unidimensionalidade concebida como parâmetro pelo modelo da TRI, foram realizadas análises dos fatores da EPSUS-A de forma separada. Cada fator da escala foi considerado como uma dimensão independente, mesmo que estivesse relacionado com o construto "suporte social" como um todo. Foi utilizado o programa estatístico Winsteps para estimar os parâmetros das pessoas (índice $\theta$, infit e outfit) e dos itens (parâmetro b, infit e outfit).

Quanto aos valores infit e outfit, foram empregados os postulados da literatura de que tais índices são adequados quando se encontram entre 0,70 e 1,30 (Linacre \& Wright, 1994; Valentini \& Laros, 2011). Além disso, foram analisados os índices de correlação, fidedignidade, categorias de respostas e mapas de itens por dimensão da EPSUS-A.

\section{Resultados e Discussão}

O parâmetro foi obtido por meio da TRI para o modelo da escala graduada para a EPSUS-A. Na Tabela 1 estão sumarizadas as estatísticas descritivas dos traços latentes (theta) dos respondentes, bem como seus respectivos índices de ajuste $\theta$ em cada um dos fatores da EPSUS-A. Além disso, essa Tabela sumariza os dados descritivos para os itens, isto é, o nível de dificuldade, os índices de ajuste, a correlação item- $\theta$, os índices de fidedignidade $\theta$ (real e modelado, linha superior), e os índices de fidedignidade dos itens (real e modelado, linha inferior).

No geral, o nível médio do traço latente nas escalas sugere que os itens tenderam a serem endossados pela amostra, uma vez que em todas as dimensões da EPSUS-A a média $\theta$ foi positiva. $O$ fator dois, Interações Sociais, foi o que apresentou a menor média de $\theta(0,6)$, indicando que seus itens foram os menos endossados pelos participantes. Já o primeiro fator, Afetivo, apresentou maior média $\theta(1,4)$, sendo os seus itens os mais endossados pela amostra.

Ainda em relação aos participantes, foram verificados os índices de ajuste por meio de infit e 
outfit, para a estimação dos thetas dos respondentes. De acordo com Linacre e Wright (1994), os valores de ajustes são considerados adequados quando os índices infit e outfit estão entre 0,7 e 1,3. Como pode ser observado na Tabela 1, os valores médios estão adequados, variando entre 0,9 e 1,0. Entretanto, no que tange às pontuações máxima (variando entre 4,0 e 4,9) e mínima $(0,0$ e $0,1)$, pode-se constatar que houve discrepâncias, para além do esperado pelo modelo, para alguns sujeitos.

Além disso, o índice de fidedignidade das estimativas de $\theta$, calculado pelo modelo de Rasch, variou entre 0,66 e 0,88 (real) e 0,73 e 0,90 (modelado). Apenas a dimensão dois, Interações sociais, apresentou parâmetro de confiabilidade abaixo de 0,70 , considerado satisfatório de acordo com a literatura (Pasquali,1999).

Em relação aos dados descritivos para os itens, o índice de dificuldade (b) apresentou variabilidade de -0,6 a 0,8, que são o mínimo e o máximo, respectivamente, do terceiro fator (Instrumental) da EPSUS-A. A média dos índices de ajuste de todas as dimensões da EPSUS-A foi adequada (entre 0,7 e 1,3), em conformidade com o que relatam Linacre e Wright (1994). Além disso, é possível verificar que não somente a média de ajuste esteve adequada, mas também os índices máximos de infit e outfit se apresentaram abaixo desse parâmetro, o que implica dizer que os itens também estão adequados.
No que se refere às correlações item-theta, elas se situaram entre 0,59 e 0,73, o que implica, de acordo com Dancey e Reidy (2006), que as correlações podem ser classificadas como de magnitude moderada $(0,40-0,69)$ e fortes $(0,70-0,99)$. Outra análise realizada por fatores foi em relação aos dados das categorias de resposta. Esse tipo de análise fornece a distribuição das quatro possíveis respostas da EPSUS-A em relação a dois eixos, horizontal (eixo x) e vertical (eixo y). Ao centro, encontra-se a distribuição de categorias de respostas da EPSUS-A: "Nunca" (0), "Poucas vezes" (1), "Muitas vezes" (2) e "Sempre" (3). A intersecção entre duas categorias é interpretada como o valor limiar (threshold) de transição entre as categorias.

Nessa mesma direção, o fator 1 , Afetivo, apresentou, no eixo $x$, horizontal, a escala $\theta$, variando de -4 a 4 . Por sua vez, o eixo y, vertical, variou de 0,0 a 1,0, representando a probabilidade de resposta dos participantes nos diferentes níveis de $\theta$. Em relação ao primeiro fator da EPSUS-A, O threshold entre as categorias 0 e 1 foi de $-1,88$; entre a 1 e 2 , igual a $-0,23$; e entre 2 e 3, de 2,11. Dado que para esse tipo de análise são esperadas separações das curvas em diferentes regiões na escala de $\theta$ (eixo horizontal), isso representa que as respostas aos itens foram modeladas quantitativamente por meio de uma relação monotônica crescente entre $\theta$ e a categoria escalar.

Tabela 1

Estatísticas descritivas sumarizadas das pessoas e dos itens

\begin{tabular}{|c|c|c|c|c|c|c|c|c|c|}
\hline & & \multicolumn{4}{|c|}{ Pessoas } & \multicolumn{3}{|c|}{ Itens } & \multirow[b]{2}{*}{ Fidedignidade } \\
\hline & & $\theta$ & Infit & Outfit & $b$ & Infit & Outfit & Correlação & \\
\hline \multirow[t]{3}{*}{ Fator 1} & $X(D P)$ & $1,4(1,3)$ & $1,0(0,5)$ & $1,0(0,5)$ & $0,0(0,2)$ & $1,0(0,0)$ & $1,0(0,0)$ & $0,60-0,67$ & $0,88(0,90)$ \\
\hline & Máximo & 4,9 & 4,0 & 4,1 & 0,4 & 1,1 & 1,1 & & $0,89(0,89)$ \\
\hline & Mínimo & $-2,9$ & 0,0 & 0,0 & $-0,4$ & 0,8 & 0,7 & & \\
\hline \multirow[t]{3}{*}{ Fator 2} & $X(D P)$ & $0,6(1,3)$ & $1,0(0,7)$ & $1,0(0,7)$ & $0,0(0,4)$ & $0,9(0,1)$ & $1,0(0,1)$ & $0,66-0,73$ & $0,66(0,73)$ \\
\hline & Máximo & 3,5 & 4,9 & 5,0 & 0,6 & 1,2 & 1,2 & & $0,98(0,98)$ \\
\hline & Mínimo & $-3,6$ & 0,1 & 0,1 & $-0,5$ & 0,8 & 0,8 & & \\
\hline \multirow[t]{3}{*}{ Fator 3} & $X(D P)$ & $0,8(1,2)$ & $1,0(0,6)$ & $0,9(0,6)$ & $0,0(0,4)$ & $0,9(0,1)$ & $0,9(0,1)$ & $0,59-0,72$ & $0.73(0.77)$ \\
\hline & Máximo & 3,4 & 4,5 & 5,0 & 0,8 & 1,1 & 1,2 & & $0.98(0.98)$ \\
\hline & Mínimo & $-3,2$ & 0,0 & 0,0 & $-0,6$ & 0,8 & 0,8 & & \\
\hline \multirow[t]{3}{*}{ Fator 4} & $X(D P)$ & $0,8(4,0)$ & $1,0(0,7)$ & $1,0(0,7)$ & $0,0(0,2)$ & $0,9(0,1)$ & $1,0(0,1)$ & $0,66-0,73$ & $0.76(0.81)$ \\
\hline & Máximo & 4,0 & 4,5 & 4,5 & 0,3 & 1,1 & 1,1 & & $0.89(0.90)$ \\
\hline & Mínimo & $-3,9$ & 0,0 & 0,0 & $-0,3$ & 0,8 & 0,8 & & \\
\hline
\end{tabular}


Nos dados das categorias de respostas do fator 2, Interações sociais, o valor limiar entre as categorias 0 e 1 foi de -2,09; entre a 1 e 2, igual a $-0,09$; e entre 2 e 3, o threshold foi de 2,00. Quanto à distribuição das categorias de respostas, também houve separação das curvas em diferentes regiões na escala de $\theta$ (eixo horizontal) e categorias crescentes ao longo da escala $\theta$.

Em relação ao fator 3, instrumental, o valor limiar entre as categorias 0 e 1 foi de -1,31; entre a 1 e 2 , igual a -0,14; e entre 2 e 3 , o threshold foi de 1,45 . Semelhante ao que foi visto nos fatores $1 \mathrm{e}$ 2, a dimensão Instrumental da EPSUS-A também apresentou separação das curvas de resposta em diferentes regiões na escala theta, assim como também é possível observar categorias crescentes ao longo da escala theta.

Já no fator 4, Enfrentamento de problemas, foi possível verificar que o valor limiar entre as categorias 0 e 1 foi de $-2,11$; entre a 1 e 2 , foi igual a -0,12; e entre 2 e 3, o threshold foi de 2,23. Quanto à distribuição das categorias de respostas, assim como os demais fatores da EPSUS-A, também houve separação das curvas em diferentes regiões na escala de $\theta$ (eixo horizontal) e categorias crescentes ao longo da escala $\theta$. Em outras palavras, percebeu-se que os itens da EPSUS-A estiveram adequados ao modelo de resposta adotado, com quatro opções.

Fazendo uso da TRI, de acordo com Embretson (2006), é possível realizar normatização com referência ao item, ou seja, verificar os significados para as pontuações dos sujeitos da amostra em referência aos itens do instrumento, em particular para este estudo, realizado por meio de mapas de itens das dimensões da EPSUS-A, conforme ilustram as Figuras 1, 2, 3 e 4. Como forma de direcionar o leitor, o símbolo " $¥$ " no mapa representa 6 pessoas, a letra " $\mathrm{M}$ " (localizada tanto no lado dos itens como no das pessoas) representa a média, " $S$ " significa um desvio-padrão, " $T$ " representa dois desvios-padrão, e o ponto "." significa um número entre 1 e 5 pessoas.

Com base na análise da Figura 1 pode-se constar que a dificuldade dos itens (lado direito do mapa de itens) esteve entre -1 e +1 , enquanto o $\theta$ das pessoas (lado esquerdo do mapa de itens) esteve entre -3 e +5 . A média dos itens foi inferior à média das pessoas, representando que os itens foram facilmente respondidos pelas pessoas da amostra utilizada. No que tange à facilidade ou dificuldade dos itens, o item 33 "Confiam em mim" foi o mais fácil de ser respondido, enquanto o item 28 "Me elogiam" foi o que apresentou maior dificuldade de resolução.

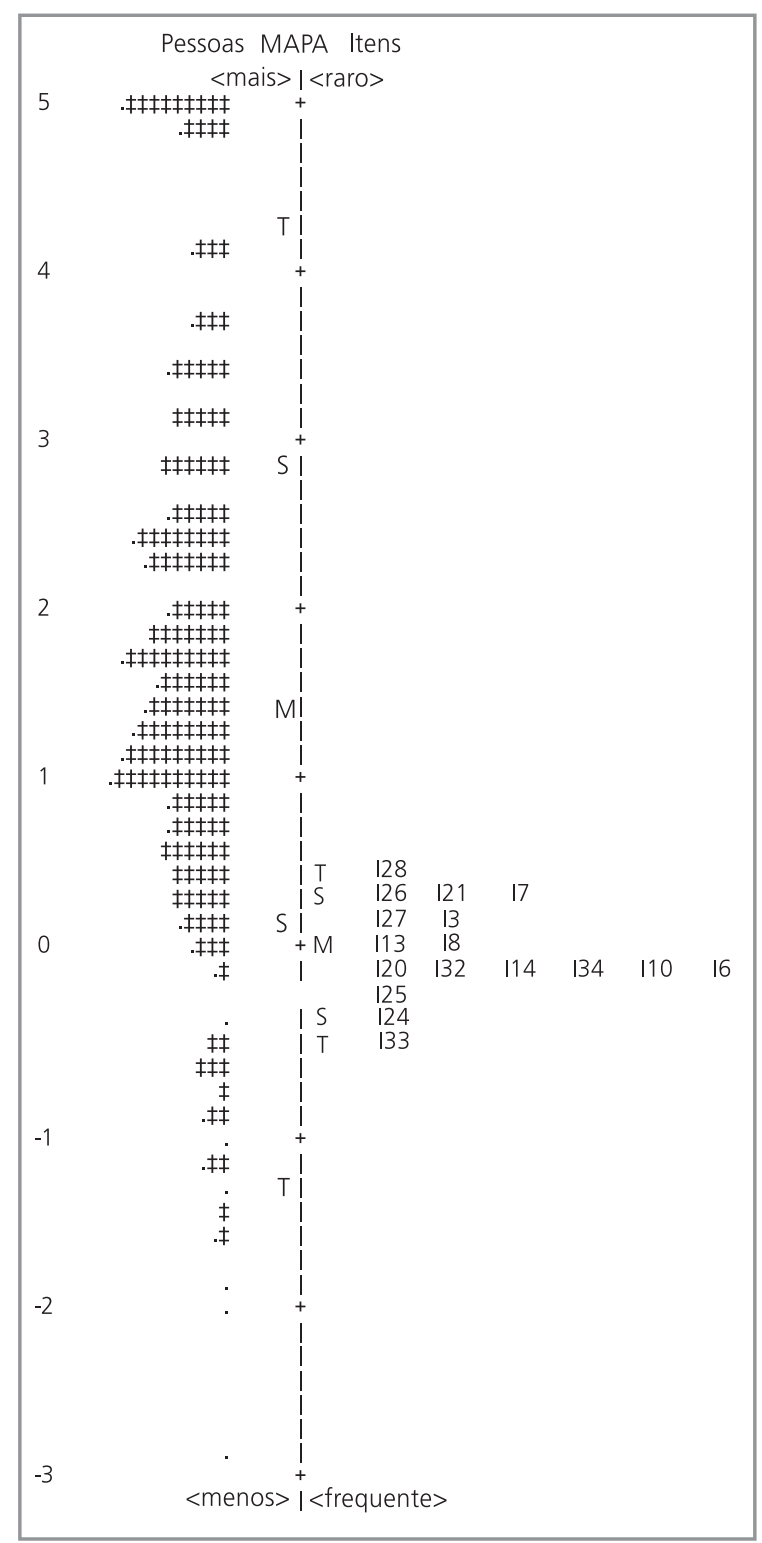

Figura 1. Mapa de itens da dimensão Afetivo da Escala de Percepção do Suporte Social-versão Adulta.

Nota: ¥: Corresponde a 6 pessoas; M: Média; S: Um desvio-padrão; T: Dois desvios-padrão; .: entre 1 a 5 pessoas. 


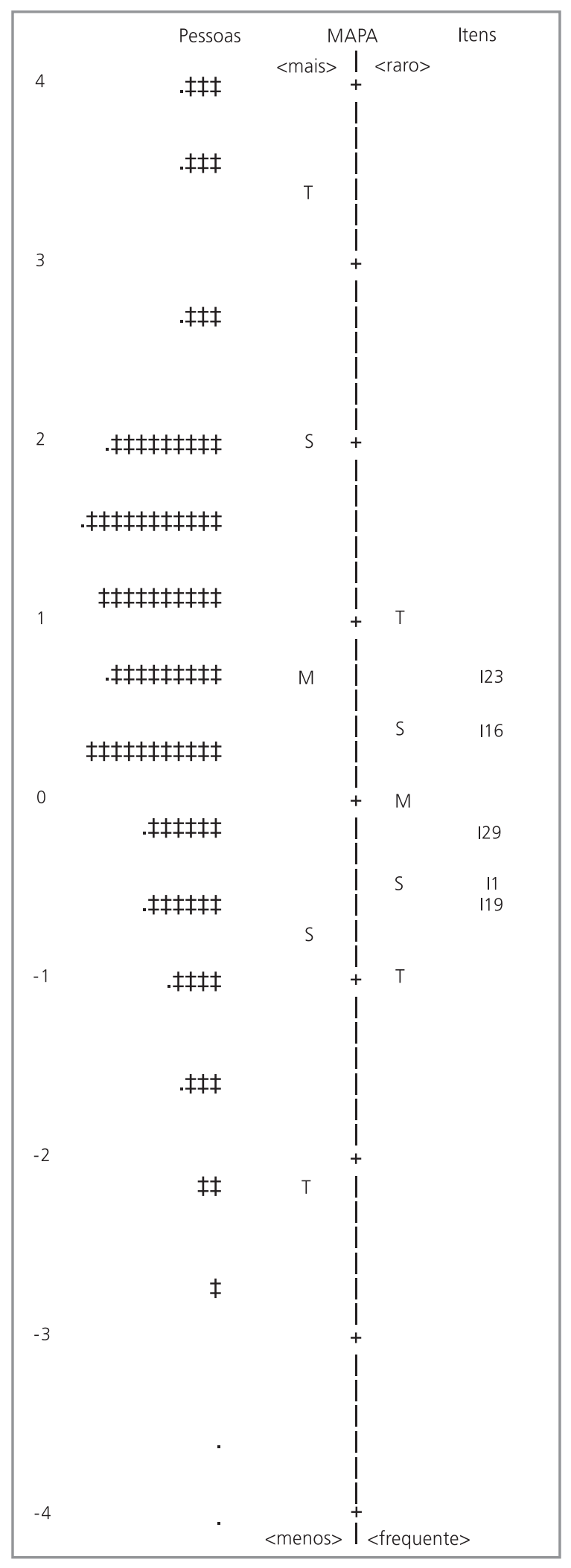

Figura 2. Mapa de itens da dimensão Interações Sociais da Escala de Percepção do Suporte Social-versão Adulta.

Nota: ł: Corresponde a 6 pessoas; M: Média; S: Um desvio-Padrão; T: Dois desvios-padrão; .: entre 1 a 5 pessoas.

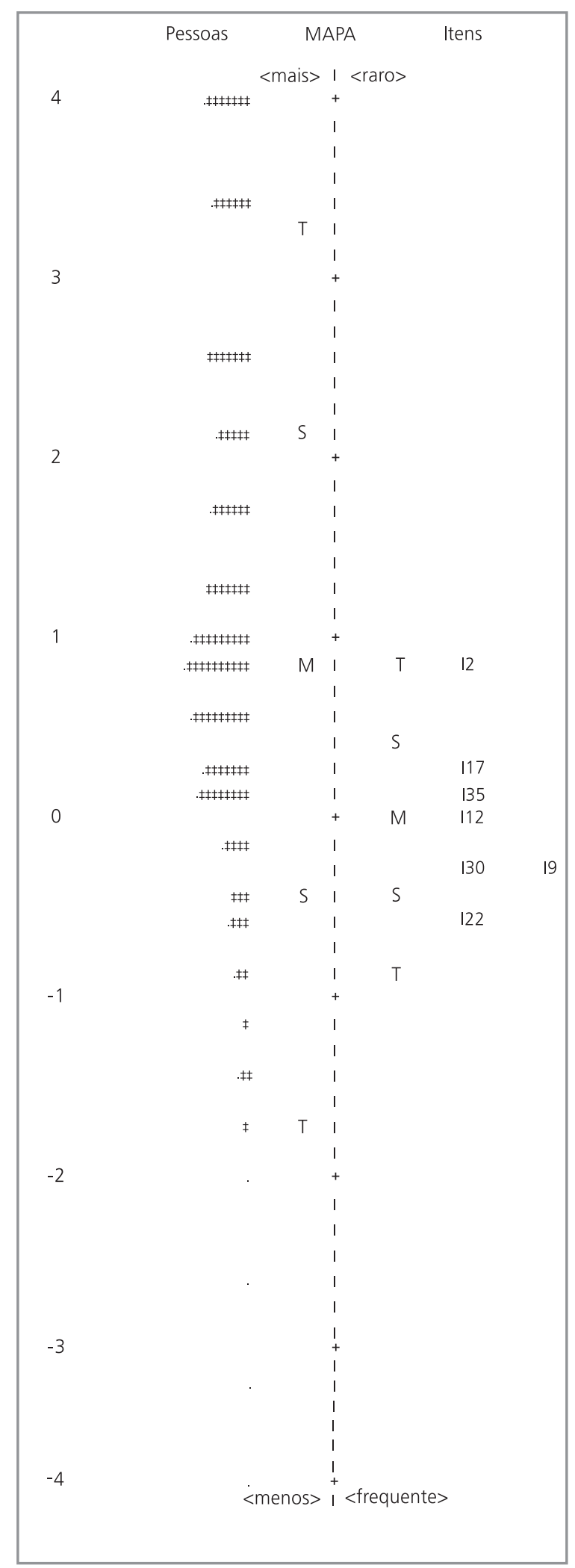

Figura 3. Mapa de itens da dimensão Instrumental da Escala de Percepção do Suporte Social-versão Adulta.

Nota: ¥: Corresponde a 6 pessoas; M: Média; S: Um desvio-padrão; T: Dois desvios-padrão; .: entre 1 a 5 pessoas. 


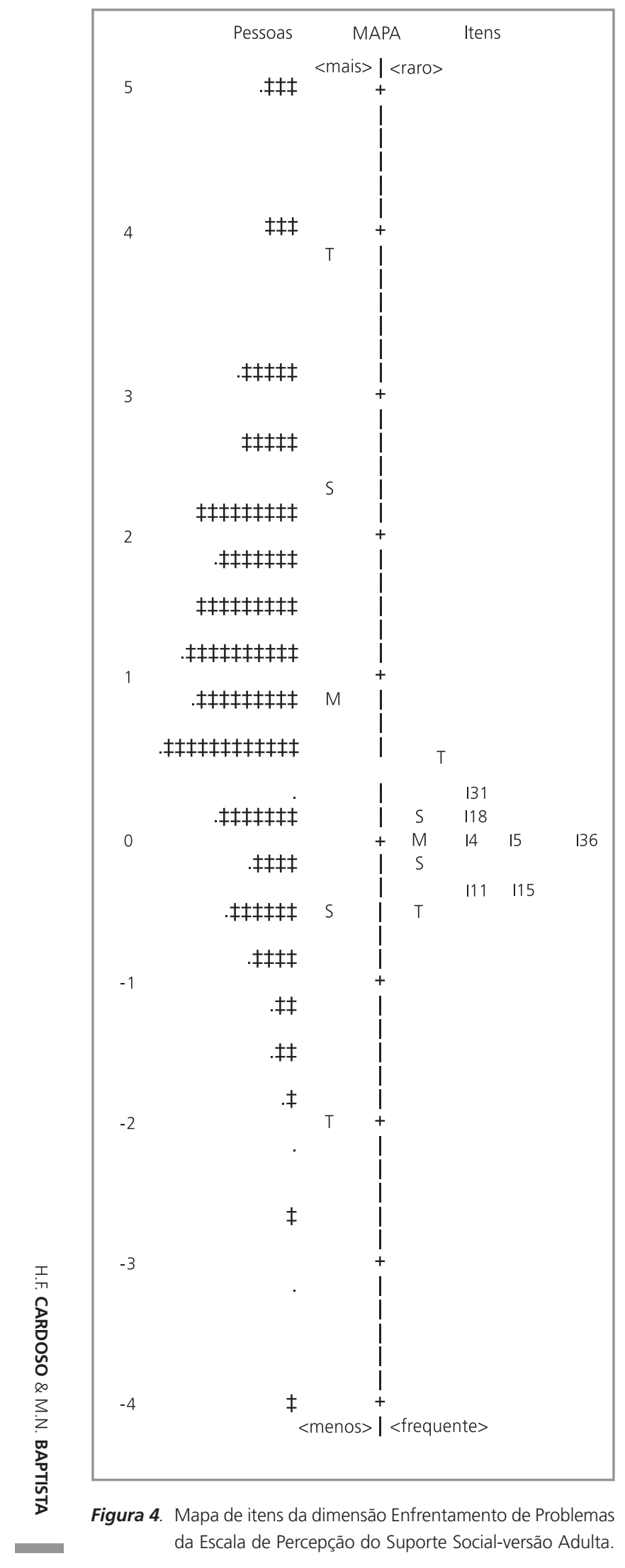

Nota: ¥: Corresponde a 6 pessoas; M: Média; S: Um desvio-padrão; T:

474

Dois desvios-padrão; .: entre 1 a 5 pessoas.
A Figura 2 permite indagar que a dificuldade dos itens esteve entre -1 e +1 , enquanto o theta das pessoas esteve entre -4 e +4 . Assim como no fator 1, a média dos itens foi inferior à média das pessoas e, quanto aos itens, o de número 19 "Me convidam para atividades de lazer" foi o mais facilmente endossado pela amostra, enquanto o de número 23 "Entendem meus valores" foi o que apresentou maior dificuldade de endosso.

Na Figura 3 é possível observar que a dificuldade dos itens esteve entre -1 e +1 , enquanto $o$ theta esteve entre -4 e +4 . A média dos itens foi inferior à média das pessoas, sendo que o item 2 se apresentou em um mesmo nível da média das pessoas. No que tange à facilidade ou dificuldade dos itens, o de número 22 "Me ajudam caso venha a ficar doente" foi o mais fácil de ser aderido, enquanto o de número 2 "Pagam minhas contas quando tenho um problema financeiro" foi o mais endossado.

Com base na análise da Figura 4, pode-se constar que a dificuldade dos itens esteve entre -1 $e+1$, enquanto $o$ theta das pessoas esteve entre -4 e +5 . A média dos itens foi inferior à média das pessoas, representando que os itens foram facilmente respondidos pelas pessoas da amostra. Em relação à facilidade ou dificuldade dos itens, o número 11 "Auxiliam em momentos de tomadas de decisões em minha vida" e o 15 "Me auxiliam a compreender determinada situação" foram os mais facilmente endossados, enquanto o número 31 "Discutem meus problemas" foi o item de maior dificuldade de resolução pelas pessoas.

Em linhas gerais, foi possível perceber que, embora os itens da EPSUS-A tenham sido endossados facilmente pela amostra, uma vez que em todas as dimensões a média dos itens foi inferior à média theta, nos fatores Interações Sociais e Instrumental, os itens mais difíceis estiveram em um mesmo parâmetro da média $\theta$.

\section{Considerações Finais}

A presente pesquisa teve como propósito a análise de parâmetros, por meio do modelo de Rasch, para a EPSUS-A. Assumindo-se o parâmetro 
da unidimensionalidade, optou-se por analisar os quatro fatores do instrumento em separado. Como pôde ser observado, os valores encontrados foram adequados ao modelo adotado. No caso específico da EPSUS-A, que já possui estudos de evidências de validade com base na estrutura interna e na relação com outras variáveis, o modelo da TRI foi utilizado visando adequações de itens, o que resultou em resultados satisfatórios, uma vez que não houve nenhum item que tenha se apresentado como disfuncional.

Por fim, vale ressaltar que este estudo apresenta limitações relacionadas à sua amostra, já que essa foi composta por universitários que, pelo menos em tese, tendem a apresentar adequada percepção do suporte social - em detrimento de outras amostras. Para estudos futuros, sugere-se uma amostra mais heterogênea, a fim de verificar se os itens se mantêm dentro dos parâmetros adequados ou se há variabilidade nos valores.

\section{Referências}

Andrade, D. F., \& Valle, R. C. (1998). Introdução à teoria de resposta ao item. Estudos em Avaliação Educacional, 18, 13-32.

Baptista, M. N., \& Cardoso, H. F. (2012). Escala de Percepção do Suporte Social (versão adulto) - EPSUS-A: (Relatório técnico não-publicado). Universidade São Francisco, Itatiba.

Cardoso, H. F. (2013). Construção e estudos psicométricos da Escala de Percepção do Suporte Social (versão adulta) EPSUS-A (Tese de doutorado não-publicada). Universidade São Francisco, Itatiba.

Carvalho, L. F. (2011). Desenvolvimento e verificação das propriedades psicométricas do inventário dimensional clínico da personalidade (Tese de doutorado não-publicada). Universidade São Francisco, Itatiba.

Carvalho, L. F., \& Primi, R. (2010). Development of a Brazilian inventory for the assessment of personality disorders based on Millon's model. Poster session presented at the annual meeting of the Society for Personality Assessment, California.

Comrey, A. L., \& Lee, H. B. (1992). A first coursefor identifying biased test items. Hillsdale: Erlbaum.

Conde, F. N., \& Laros, A. J. (2007). Unidimensionalidade e a propriedade de invariância das estimativas da habilidade pela TRI. Avaliação Psicológica, 6(2), 205-215.
Dancey, C. P., \& Reidy, J. (2006). Estatística sem matemática para psicologia usando o SPSS para Windows ( $3^{a}$ ed.). Porto Alegre: Artmed.

Embretson, S. E., \& Reise S. P. (2000). Item response theory for psychologists. Mahwah (NJ): Lawrence Erlbaum.

Embretson, S. E. (2006). The continued search for nonarbitrary metrics in psychology. American Psychologist, 61(1), 50-55.

Hambleton, R. K., \& van der Linden, W. J. (1982). Advances in item response theory and applications: An introduction. Applied Psychological Measurement, 6, 373-378.

Hambleton, R., Swaminathan, H. \& Rogers, J. (1991). Fundamentals of item response theory. New York: Sage.

Linacre, J. M. (2009). WINSTEPS: Multiple-choice, rating scale, and partial credit Rasch analysis. Chicago: MESA Press.

Linacre, J. M., \& Wright, B. D. (1994). Reasonable meansquare fit values. Rasch Measurement Transactions, 8(3), 370.

Muñiz, J. (2010). Las teorias de los tests: teoría clássica y teoría de respuesta a los ítems. Papeles del Psicólogo, 31(1), 57-66.

Nunes, C. H. S. S., \& Primi, R. (2005). Impacto do tamanho da amostra na calibração de itens e estimativa de escores por teoria de resposta ao item. Avaliação Psicológica, 4(2), 141-153.

Pasquali, L. (1999). Instrumentos psicológicos: manual prático de elaboração. Brasília: LabPAM.

Pasquali, L. (2007). Teoria de resposta ao item. Brasília: Universidade de Brasília.

Pasquali, L. \& Primi, R. (2003). Fundamentos da Teoria de Resposta ao Item - TRI. Avaliação Psicológica, 2(2), 99-110.

Pasquali, L., \& Primi, R. (2007). Fundamentos da Teoria da Resposta ao Item - TRI. In L. Pasquali (Org.), Teoria de Resposta ao Item: TRI (pp.11-28). Brasília: Universidade de Brasília.

Primi, R. (2004). Avanços na interpretação de escalas com a aplicação da teoria de resposta ao item. Avaliação Psicológica, 3(1), 53-58.

Reise, S., Ainsworth, A., \& Haviland, M. (2005). Item response theory. Current Directions in Psychological Science, 14(2), 95-101.

Valentini, F., \& Laros, J. A. (2011). Teoria de resposta ao item na avaliação psicológica. In R. A. M. Ambiel, I. S. Rabelo, S. V. Pacanaro, G. A. S. Alves, \& I. F. A. S. Leme. Avaliação psicológica: guia de consulta para estudantes e profissionais de psicologia (pp.7-30). São Paulo: Casa do Psicólogo. 
Vendramini, C. M. M. (2005). Aplicação da teoria de resposta ao item na avaliação educacional. In R. Primi (Org.), Temas em avaliação psicológica (pp.229-254). São Paulo: Casa do Psicólogo.

Vieira, M. J., Ribeiro, R. B., Almeida, L., \& Primi, R. (2011). Comparação de modelos da Teoria de Resposta ao Item (TRI) na validação de uma prova de dependência-independência de campo. Avaliação Psicológica, 10(1), 63-70.

Werlang, B. S. G., Villemor-Amaral, A. E., \& Nascimento, R. S. G. F. (2010). Avaliação psicológica, testes e possibilidade de uso. In Conselho Federal de Psicologia.
Avaliação psicológica: diretrizes na regulamentação da profissão. Brasília: Conselho Federal de Psicologia.

Wiberg, M. (2004). Classical test theory vs. Item response theory: An evaluation of the theory test in the Swedish driving-license test. (50). Retrieved December 10, 2012 , from http://www.umu.se/edmeas//publikationer/pdf/ EM\%20no\%2050.pdf

Recebido em: 22/5/2013

Versão final em: 2/8/2013

Aprovado em: 13/9/2013 